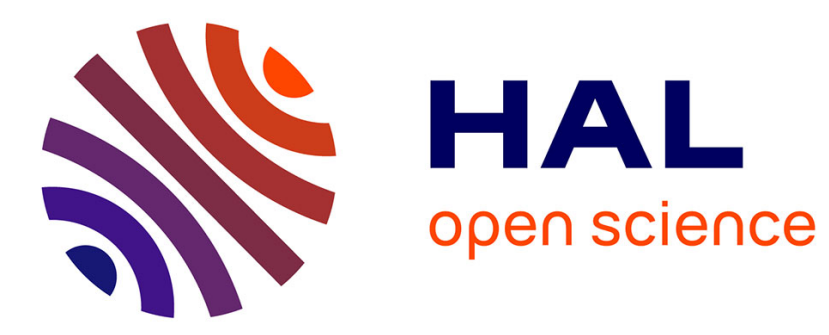

\title{
Étude par équations intégrales d'une éprouvette C.T. 15
}

\author{
J.M. Boissenot, J.C. Lachat, J. Watson
}

\section{To cite this version:}

J.M. Boissenot, J.C. Lachat, J. Watson. Étude par équations intégrales d'une éprouvette C.T. 15. Revue de Physique Appliquée, 1974, 9 (4), pp.611-615. 10.1051/rphysap:0197400904061100 . jpa00243825

\section{HAL Id: jpa-00243825 https://hal.science/jpa-00243825}

Submitted on 1 Jan 1974

HAL is a multi-disciplinary open access archive for the deposit and dissemination of scientific research documents, whether they are published or not. The documents may come from teaching and research institutions in France or abroad, or from public or private research centers.
L'archive ouverte pluridisciplinaire HAL, est destinée au dépôt et à la diffusion de documents scientifiques de niveau recherche, publiés ou non, émanant des établissements d'enseignement et de recherche français ou étrangers, des laboratoires publics ou privés. 


\title{
ÉTUDE PAR ÉQUATIONS INTÉGRALES D'UNE ÉPROUVETTE C. T. 15
}

\author{
J. M. BOISSENOT, J. C. LACHAT et J. WATSON
}

Département D. T. E.-Cetim, Senlis, France

\begin{abstract}
Résumé. - La technique des équations intégrales est utilisée pour déterminer le champ des tensions et des déplacements dans une éprouvette C. T. 15 en acier 35 NCD 16. Le calcul de l'intégrale $J$ de Rice permet de déterminer dans le cadre de la mécanique linéaire de la rupture le facteur d'intensité de contraintes pour plusieurs longueurs de fissure et ces valeurs sont comparées à celles obtenues à partir d'essais ou de calcul par éléments finis.
\end{abstract}

Abstract. - The boundary integral equation method is used to determine the stress and displacement fields in a C. T. 15 specimen in steel $35 \mathrm{NCD} 16$. The integral $J$ of Rice is used to calculate according to the linear theory of fracture mechanics the stress intensity factor for several crack lengths. These values are compared with experimental and finite element results.

1. Introduction. - La méthode des équations intégrales consiste en la transformation des équations d'état qui décrivent le comportement des fonctions inconnues à l'intérieur et sur le contour d'un domaine, en une équation intégrale reliant les inconnues et certaines de leurs dérivées aux valeurs qu'elles prennent sur la frontière. Pour une structure travaillant dans le domaine élastique, soumise à des conditions aux limites portant sur les déplacements et les tensions sur son contour, cette équation intégrale apparaît comme une relation entre les tensions et les déplacements inconnus aux valeurs qu'elles prennent en surface.

$\mathrm{La}$ résolution fournit directement les déplacements et les tensions inconnus sur le contour et par une relation simple ces informations en tout point intérieur à la structure. D'un point de vue plus général, cette technique permet l'étude d'une pièce mécanique à partir de son comportement en surface : le problème est ainsi diminué d'une dimension ce qui simplifie et allège la mise en donnée ou l'exploitation des résultats. Le traitement numérique du problème ainsi posé conduit à une discrétisation de la surface comme il sera indiqué plus loin et présente l'avantage sur d'autres techniques comme celle des éléments finis par exemple, de ne pas introduire de points supplémentaires à l'intérieur du domaine.

Historiquement, les méthodes par équations intégrales sont un développement de la méthode de Fredholm qui consiste en l'application de la théorie $\mathrm{du}$ potentiel en conjonction avec celle des équations intégrales linéaires. Les premières applications à l'élasticité ont été faites par Fredholm. Plus récemment, Cruse [1] aux Etats-Unis a développé des programmes de calcul sur ordinateur pour traiter les problèmes élastiques plans et tridimensionnels. Il a utilisé cette technique pour l'analyse tridimensionnelle d'une éprouvette de mécanique de la rupture [2]. Le même auteur a étendu la formulation des équations intégrales aux domaines élasto-dynamique et élasto-plastique [3], [4], pour des corps anisotropes et des matériaux compressibles avec écrouissage. Les travaux effectués au CETIM ont porté sur la généralisation [5] de la théorie permettant de transformer une équation aux dérivées partielles de type elliptique en une équation intégrale et sur l'étude de techniques numériques de résolution pouvant notamment prendre en compte des surfaces courbes ou des variations plus élaborées des déplacements et des tensions. Le programme de calcul des pièces planes, de forme quelconque, travaillant en élasticité plane, a été utilisé pour déterminer le champ des déplacements et des tensions dans une éprouvette C. T. 15 de mécanique de la rupture (acier 35 NCD 16). Le but de cet article est de montrer comment un tel programme est à même de représenter le champ des contraintes au voisinage d'une fissure pour en déterminer le facteur d'intensité de contraintes à partir de l'intégrale $J$ de Rice.

2. Formulation du problème élastique en équation intégrale. - Dans la suite, nous ne traiterons que les problèmes élastiques plans pour des milieux homogènes et isotropes, sans force de volume. Le comportement d'un domaine $V$ (Fig. 1) limité par un contour $S$ sur lequel sont imposés des tensions $p_{i}$ et des déplacements $q_{i}$ sous les hypothèses restrictives précédentes est défini par l'équation de Navier :

$$
\frac{1}{1-2 v} u_{i, i j}+u_{j, i i}=0 \quad(i, j=1,2)
$$




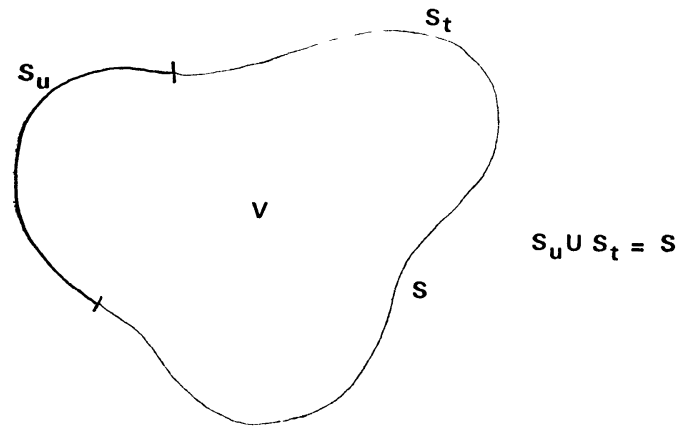

Fig. 1.

où $u_{i}(x)$ représente le déplacement du point $x$ de coordonnées cartésiennes $x_{1}, x_{2}$;

$v$ est le coefficient de Poisson.

A cette équation, sont associées les conditions aux limites suivantes :

$$
u_{i}(x)=q_{i} \quad x \in S_{u}
$$

portion du contour sur lequel les déplacements sont imposés

$$
t_{i}(x)=\sigma_{i j} n_{j}=p_{i} \quad x \in S_{t}
$$

portion du contour sur lequel les tensions sont imposées $n_{j}$ représente les composantes du vecteur normal extérieur

$\sigma_{i j}$ les composantes du tenseur des contraintes.

Une méthode générale est donnée dans l'article de Dubois et Lachat [5] pour transformer ce problème différentiel en une équation intégrale qui relie les déplacements $u_{i}$ et les tensions $t_{i}$ en surface :

$$
\begin{aligned}
\frac{1}{2} u_{i}(x)=\int_{S} \mathbf{U}_{i j}(x, \xi) t_{j}(\xi) \mathrm{d} S(\xi)- \\
\quad-\int_{S} \mathbf{T}_{i j}(x, \xi) u_{j}(\xi) \mathrm{d} S(\xi)
\end{aligned}
$$

$x$ et $\xi$ sont des points appartenant à $S$.

$\mathbf{U}$ et $\mathbf{T}$ sont des tenseurs du second ordre, singuliers pour $x=\xi$ qui correspondent aux tractions et aux déplacements pour un milieu bidimensionnel infini soumis à un effort concentré unité. Ces expressions sont données dans la référence [5].

On montre l'existence et l'unicité de la solution ainsi obtenue sous certaines conditions physiquement évidentes :

- le domaine est en équilibre,

- pas de déplacement d'ensemble.

La résolution de l'équation intégrale précédente nous donne les tensions et les déplacements inconnus sur le contour et les relations suivantes permettent d'obtenir les contraintes et les déplacements, en tous points $x$ intérieurs, c'est-à-dire appartenant à $V$, pour $x \in V, \xi \in S$

$$
\begin{aligned}
u_{i}(x)=\int_{S} \mathbf{U}_{i j}(x, \xi) t_{j}(\xi) \mathrm{d} S(\xi)- & \\
& -\int_{S} \mathbf{T}_{i j}(x, \xi) u_{j}(\xi) \mathrm{d} S(\xi) \\
\sigma_{i j}(x)=\int_{S} \mathbf{D}_{k i j}(x, \xi) t_{k}(\xi) \mathrm{d} S(\xi)- & \\
& -\int_{S} \mathbf{S}_{k i j}(x, \xi) u_{k}(\xi) \mathrm{d} S(\xi)
\end{aligned}
$$

où $\mathbf{D}_{k i j}$ et $\mathbf{S}_{k i j}$ sont les composants d'un tenseur du troisième ordre explicité dans la référence [5].

3. Le programme par équations intégrales du CETIM. - Le problème étant posé sous la forme de l'équation intégrale $(2)$; il faut essayer de se ramener à la résolution d'un système linéaire. Dans ce but, Cruse [1] propose de discrétiser le contour du domaine $V$ en un certain nombre de segments sur lesquels les déplacements et les tensions auront des valeurs constantes. Nous sommes conduits à un système linéaire dont les inconnues sont les tensions et les déplacements des nœuds milieux des segments et dont les coefficients sont les intégrales sur chaque segment des tenseurs $\mathbf{T}$ et $\mathbf{U}$; intégrales qui peuvent être obtenues analytiquement ou par traitement numérique. Quelques précautions doivent être prises dans la situation décrite précédemment lorsque ces deux tenseurs deviennent singuliers. Après introduction des conditions aux limites sur les $t_{i}$ et $u_{i}$ le système précédent est un système linéaire plein de $2 n \times 2 n$ équations où $n$ représente le nombre de segments nécessaires à la représentation du contour de la pièce. L'analogie avec la technique des éléments finis est évidente et le segment droit avec des déplacements constants joue le même rôle en équation intégrale que le triangle simple à trois nœuds dans la technique des éléments finis. Afin d'améliorer cette procédure, nous avons développé au CETIM des éléments de contour plus raffinés, en adoptant une représentation paramétrique pour la géométrie et pour les fonctions de variations des inconnues, similaire à celle utilisée en éléments finis dans le concept d' " isoparamétrie ». Sur chaque élément de contour, la géométrie a une représentation parabolique et les fonctions représentant la variation des tensions ou des déplacements peuvent être constantes, linéaires, quadratiques, cubiques. Ces éléments sont représentés sur la figure 2 : les points ronds sont attachés à la géométrie, les triangles correspondent aux nœuds sur lesquels les déplacements et les tensions sont interpolés. Les intégrations sont faites numériquement à l'aide d'une technique de Gauss améliorée à $2,3,4$ ou 5 points suivant la précision recherchée, et la résolution du système linéaire par la méthode d'élimination de Gauss avec un traitement par bloc, pour réduire la taille du problème en mémoire centrale. Le programme nous fournit: les tensions, les 


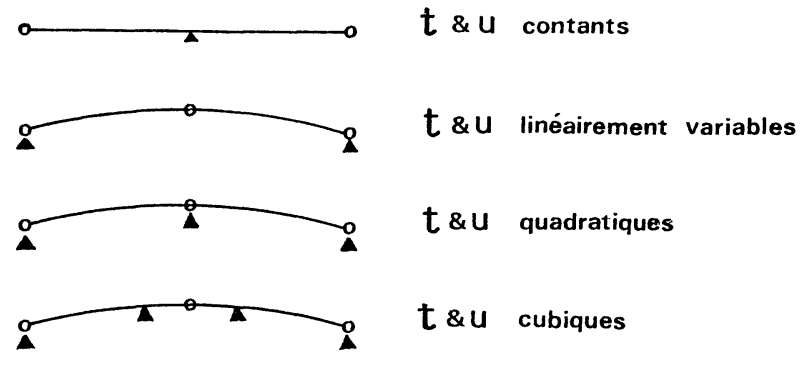

FIG. 2.

déplacements, les contraintes en tous les points du contour et les mêmes informations en tous points intérieurs définis lors de la mise en données. Cette technique des équations intégrales se caractérise:

- par une très grande souplesse et par la rapidité de la mise en données;

- par des temps de traitement pouvant être trois à quatre fois plus faibles que par la méthode des éléments finis ;

- par une grande simplicité des résultats obtenus puisque l'on peut très souvent se limiter aux informations en surface ou rechercher les déplacements et les contraintes en des points intérieurs que l'on a définis par avance. Les sorties de résultats contrastent avec les listings obtenus par éléments finis.

Ce programme est opérationnel au CETIM depuis six mois environ et il a fait l'objet de nombreuses utilisations sur des pièces planes industrielles.

4. Application à l'éprouvette C. T. 15 en acier NCD 16. - Cette éprouvette de mécanique de la rupture a été expérimentée à l'Institut de Recherche de la Sidérurgie et deux types de calcul ont été menés au Centre Technique des Industries Mécaniques, l'un aux équations intégrales, l'autre par éléments finis.

4.1 Calcul de l'éprouvette C. T. 15. - La géométrie de l'éprouvette est représentée sur la figure 3. Pour des raisons de symétrie évidente tant

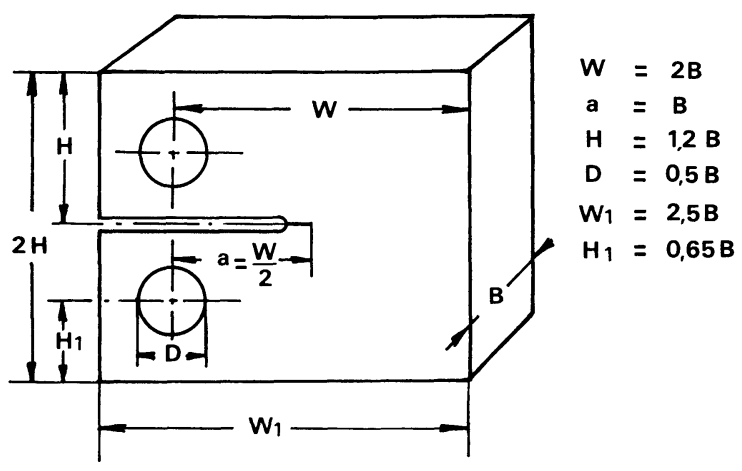

Fig. 3.

géométrique que sur les efforts appliqués, les calculs ont été conduits sur une moitié d'éprouvette en imposant les déplacements fixes dans la direction $O y$ pour le plan de fissure, sous l'hypothèse que la propagation de la fissure se fasse en ligne droite.

4.1.1 Eléments finis. - Le traitement de l'éprouvette C. T. 15 est effectué en utilisant le programme BIPLAST du CETIM qui résout, par éléments finis isoparamétriques à 12 degrés de liberté, les problèmes plans ou de révolution en régime élasto-plastique. La figure 4 représente le maillage utilisé :

- 223 éléments,

-1010 degrés de liberté.

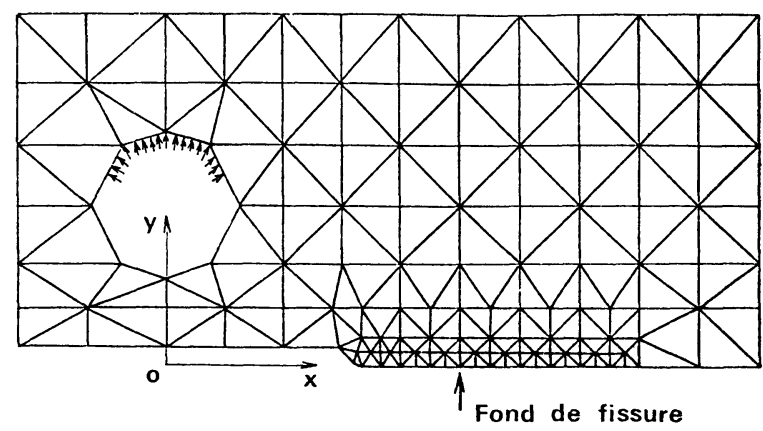

Fig. 4.

Les efforts de traction sont appliqués au sommet $\mathrm{du}$ trou. La préparation des données a nécessité environ une journée et le maillage a été prévu pour traiter plusieurs longueurs de fissures. Les calculs ont été poursuivis jusqu'au domaine élasto-plastique : l'influence de la zone plastique est faible.

4.1.2 Equations intégrales. - Dans notre programme aux équations intégrales la génération des données s'effectue automatiquement à partir d'un nombre faible de points. Le maillage représenté sur la figure 5 est obtenu à partir des points numérotés de 1 à 12 ; le trou est généré en coordonnées

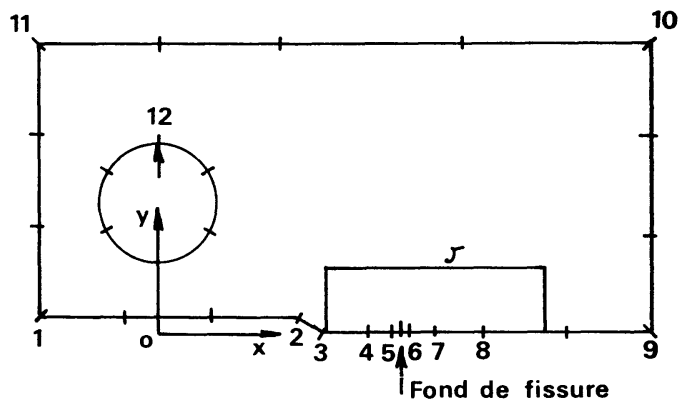

FIG. 5.

cylindriques dès que l'on connaît les coordonnées de son centre et celles du point 12 . Sur chaque élément les tensions et les déplacements ont des variations cubiques, ce qui conduit :

- à 28 éléments géométriques de type quadratique, - 168 degrés de liberté. 
Le point noté 9 est fixé pour supprimer les déplacements d'ensemble et les points du plan de fissure ne peuvent pas se déplacer dans la direction $O y$.

L'effort de traction est appliqué au point noté 12 . Les données peuvent être générées en une heure environ et le prix de revient du passage sur ordinateur est deux fois moins cher que par éléments finis.

4.2 Ouverture De Fissure. - La déformée de la fissure sous l'effet de la traction extérieure est représentée sur la figure 6. L'accord est excellent entre les calculs aux éléments finis et les résultats obtenus par équation intégrale.

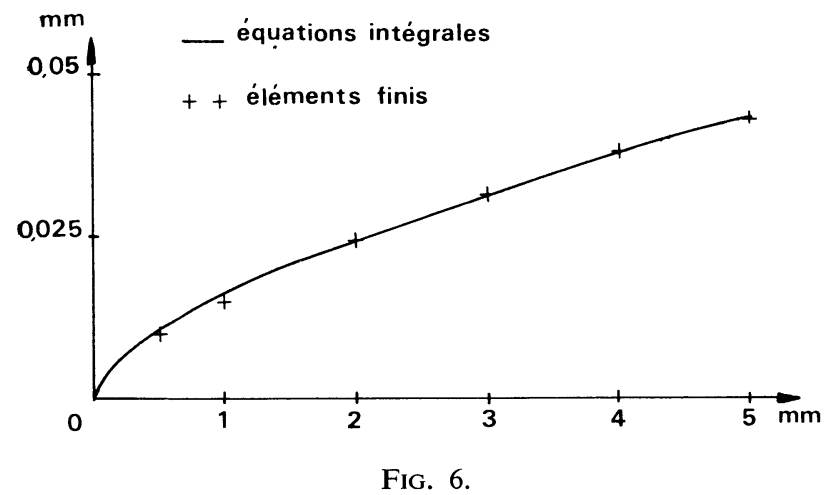

4.3 DéTERMination DU FACTEUR D'INTENSITÉ DE

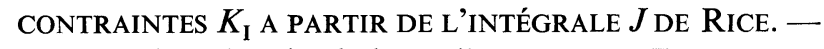
L'intégrale $J$ de Rice le long d'un contour $\Gamma$ entourant le sommet de fissure est donnée par la relation :

$$
J=\int_{\Gamma}\left(\omega \mathrm{d} y-\mathbf{T} \cdot \frac{\partial \mathbf{u}}{\partial x} \mathrm{~d} s\right)
$$

où $\omega$ représente la densité d'énergie de déformation :

$$
\omega=\int_{0}^{\varepsilon} \sigma_{i j} \mathrm{~d} \varepsilon_{i j}
$$

$\mathbf{T}$ et $\mathbf{U}$ représentent les tensions et les déplacements le long d'un contour $\Gamma$ d'abscisse curviligne $s$, entourant le sommet de fissure.

$J$ est évalué à partir des résultats obtenus par équations intégrales et par éléments finis le long du même contour (Fig. 4 et 5) pour une longueur de fissure $a / W=0,5$ (Fig. 3).

Les calculs effectués par éléments finis pour un comportement du matériau élastique parfaitement plastique ont montré que l'erreur relative sur $J$, en adoptant un comportement élastique, était inférieure à $1 \%$.

Dans le domaine élastique, la valeur de $J$ est reliée au facteur d'intensité de contraintes en mode I par la relation :

$$
J=\frac{1-v^{2}}{E} K_{\mathrm{I}}^{2} \quad \text { déformations planes . }
$$

Le tableau I donne une comparaison entre les résultats expérimentaux obtenus par l'IRSID et les calculs aux éléments finis et par équations intégrales pour le facteur d'intensité de contraintes critiques sur cinq éprouvettes C. T. 15 acier 35 NCD 16, pour

\begin{tabular}{|c|c|c|c|c|}
\hline $\begin{array}{l}\text { No des } \\
\text { éprou- } \\
\text { vettes }\end{array}$ & $P_{C}$ daN & IRSID & $\begin{array}{c}\text { KIc hbar } \times \sqrt{\mathrm{mm}} \\
\text { éléments } \\
\text { finis }\end{array}$ & $\begin{array}{l}\text { équations } \\
\text { intégrales }\end{array}$ \\
\hline - & - & - & - & 一 \\
\hline 1 & 1660 & 194 & 189 & 192 \\
\hline 2 & 1934 & 226 & 221 & 223 \\
\hline 3 & 2088 & 224 & 239 & 242 \\
\hline 4 & 2133 & 247 & 241 & 247 \\
\hline 5 & 2225 & 260 & 254 & 257 \\
\hline
\end{tabular}
une longueur de fissure $a / W=0,5$.

\section{TABLEAU I}

4.4 DÉTERMINATION DU FACTEUR D'INTENSITÉ DE CONTRAINTES A PARTIR DE LA VARIATION DE L'ÉNERGIE POTENTIELLE. - La force d'extension de fissure $G$ est reliée à la variation d'énergie potentielle par unité d'épaisseur par la relation :

$$
G=\frac{\partial \bar{U}}{\partial a}=\frac{1-v^{2}}{E} K_{\mathrm{I}}^{2}=\frac{1}{W} \frac{\partial \bar{U}}{\partial(a / W)}
$$

$U$ est l'énergie potentielle par unité d'épaisseur d'où

$$
K_{\mathrm{I}}^{2}=\frac{1}{W}\left[\frac{E}{1-v^{2}} \frac{\partial \bar{U}}{\partial(a / W)}\right]
$$

que l'on préfère mettre sous la forme :

$$
K_{\mathrm{I}}=\frac{P}{B \sqrt{\bar{W}}} Y\left(\frac{a}{W}\right)
$$

avec

$$
Y\left(\frac{a}{W}\right)=\frac{B}{P}\left[\frac{E}{1-v^{2}} \frac{\partial \bar{U}}{\partial(a / W)}\right]^{1 / 2}
$$

$P$ effort de traction, $B$ épaisseur de l'éprouvette.

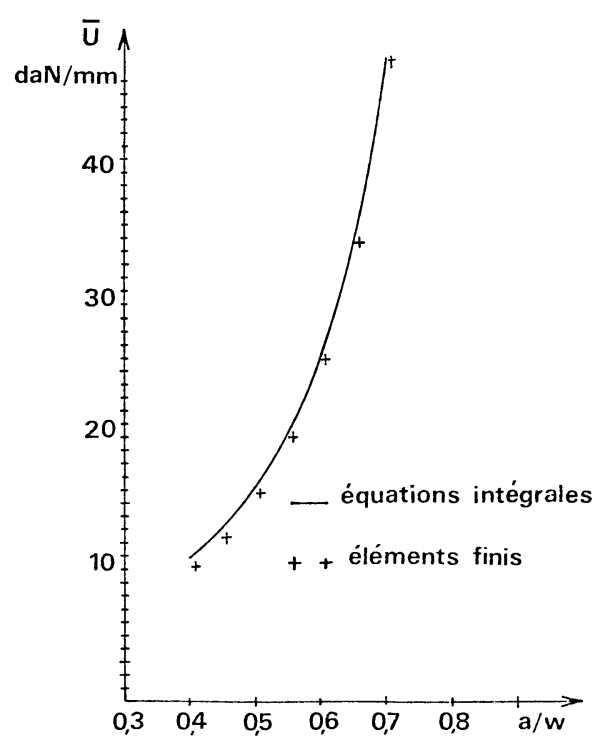

FIG. 7. 
Pour différentes longueurs de fissure, on calcule l'énergie élastique de déformation emmagasinée dans l'éprouvette; elle est égale au travail des forces extérieures.

On calcule pour une charge donnée et pour plusieurs longueurs de fissure $(a / W)$ l'énergie potentielle par unité d'épaisseur.

A partir de ces points, on construit une courbe $\bar{U}=f(a / W)$ à l'aide de fonctions splines cubiques. On détermine alors directement les dérivées premières et secondes en tous points de l'intervalle délimité par les valeurs extrêmes des longueurs de fissures (Fig. 7). Le tableau II compare, pour plusieurs longueurs de fissure, les valeurs de la fonction $Y(a / W)$

\section{TABLEAU II}

\begin{tabular}{|c|c|c|c|c|}
\hline$W$ & ASTM & $\operatorname{MEF}(G)$ & $(G)$ & EI \\
\hline 0,40 & 7,323 & 6,943 & 7,295 & 6,993 \\
\hline 0,45 & 8,337 & 8,321 & 8,253 & 8,235 \\
\hline 0,50 & 9,603 & 9,651 & 9,538 & 9,505 \\
\hline 0,55 & 11,260 & 11,183 & 11,242 & 11,161 \\
\hline 0,60 & 13,540 & 13,574 & 13,530 & 13,429 \\
\hline 0,65 & 16,778 & 16,618 & 16,523 & 16,500 \\
\hline 0,70 & 21,427 & 21,436 & 21,430 & 20,951 \\
\hline
\end{tabular}

données par l'ASTM à celles calculées par éléments finis et par équations intégrales. Pour ces dernières, deux méthodes ont été utilisées, l'une par l'intermédiaire de la force d'extension de fissure $G$, l'autre en utilisant l'intégrale de Rice sur le contour précédemment décrit.

5. Conclusion. - La technique des équations intégrales comme celle des éléments finis d'ailleurs est un outil bien adapté à la recherche du facteur d'intensité de contraintes dans le cadre de la mécanique linéaire de la rupture. Elle présente un certain nombre d'avantages sur la technique des éléments finis en ce qui concerne les entrées de données, le temps de traitement et l'exploitation des résultats ; avantage qu'il faudrait préciser sur des exemples de fissures dans des géométries plus complexes que celles d'une éprouvette. Néanmoins, ces premiers résultats nous permettent de penser que l'étude tridimensionnelle des fissures dans un corps épais pourra être abordée avec succès par cette technique.

Nous développons actuellement au CETIM un programme de calcul tridimensionnel par équations intégrales utilisant des éléments de contour sophistiqués et basés sur les mêmes principes que le programme bidimensionnel.

\section{Bibliographie}

[1] Cruse, T. A., «Numerical solutions in three dimensional elastostatics », Int. J. Solids structures 5 (1969) 1259.

[2] Cruse, T. A., "Application of the boundary integral equation method to three dimensional stress analysis", Computers structures 3 (1973) 509.

[3] CRUSE, T. A., " A direct formulation and numerical solution of the general transient elastodynamic problem », J. of Math. Analysis and Applications 22 (1968) $\mathrm{n}^{\circ} 2$.

[4] Cruse, T. A. and Swedlow, J. L., " Formulation of boundary integral equations for three dimensional elastoplastic flow », Int. J. Solids Structures 7 (1971) 1673.
[5] Dubois, M. and Lachat, J. C., The integral formulation of boundary value problems, Variational methods in engineering (University of Southampton) 1972, p. 989.

[6] Dubois, M., Introduction à la mécanique de la rupture, Note Technique du CETIM, n 4, Oct. 1973.

[7] Lachat, J. C., Application de la méthode des éléments finis à l'élasticité plane et aux corps de révolution, Mémoires Techniques du CETIM, no 9, Sept. 1971.

[8] SANZ (G.), La mécanique de la rupture et son application à l'étude de la ténacité des aciers, Bulletin du cercle d'études des métaux, tome XII, no 7, Sept. 1973. 